\title{
Transmisión del conocimiento y redes sociales en Patagonia centro-meridional, Argentina: un análisis de las habilidades de talla lítica en el Holoceno medio y tardío
}

\author{
Mariana SACCHI \\ CONICET - Instituto Nacional de Antropología y Pensamiento Latinoamericano, \\ Universidad de Buenos Aires \\ sacchi.mariana@gmail.com
}

Recibido: 3 de febrero de 2014

Aceptado: 30 de septiembre de 2016

\section{RESUMEN}

El objetivo de este trabajo es vincular la tecnología lítica y la circulación de información a través de la transmisión del conocimiento. Se utiliza como fuente de datos el análisis del material lítico de tres sitios procedentes de Patagonia Centro-Meridional, Argentina, y el análisis de material lítico experimental. Se analizan los índices de eficiencia para la talla, así como la presencia de diferentes tipos de errores que se identificaron como producto de talladores con menor experiencia. La presencia de estos tipos de materiales se relaciona con la posible presencia de redes sociales en el área analizada. Finalmente se plantea la posibilidad de identificación de los distintos sitios a partir de las actividades de talla realizadas en ellos.

Palabras clave: Tecnología lítica, redes sociales, transmisión del conocimiento, errores de talla.

Knowledge Flow and Social Networks in South Central Patagonia, Argentina: An Analysis of Flintknapping Expertise During Middle and Late Holocene

\begin{abstract}
The aim of this paper is to link lithic technology and the flow of information through the transmission of knowledge. The analysis of lithic material from three sites of Central Southern Patagonia, Argentina, and experimental analysis of lithic material are used as data source. Efficiency ratios are analyzed, as well as the presence of different types of errors that were identified as a result of less experienced knappers. The presence of these types of materials is related to the possible presence of social networks in the area analyzed. Finally, the possibility of identifying individual sites from knapping activities is developed.
\end{abstract}

Key words: Lithic technology, social networks, knowledge flow, flint knapping mistakes.

Sumario: 1. Introducción. 2. Planteamiento del problema: la circulación de información, la transmisión del conocimiento y la tecnología lítica. 3. Material experimental: red íntima y adquisición de habilidades para la talla. 4. Del material experimental al material arqueológico: resultados y discusión. 5. Consideraciones finales. 6. Referencias bibliográficas.

\section{Introducción}

El principal interés de este trabajo es la relación entre la tecnología y la circulación de información, principalmente la transmisión del conocimiento, usando como fuente de datos los brindados por el análisis del material lítico, vinculado con otras líneas de evidencia desde una perspectiva macro y micro-regional.

Se retoma el planteamiento de Gamble (1993a, 1993b), quien estudia las sociedades cazadoras-recolectoras como redes de redes en las que los individuos interactúan. En ellas se combinaría la riqueza del flujo de información con la facilidad de res- 
puesta que puede existir en la interacción cara a cara. Los individuos forman parte, desde esta perspectiva, de muchas redes que se entrecruzan en sus relaciones. Dentro de esta variedad, resultan de especial interés en este trabajo las denominadas redes íntimas y redes efectivas (Gamble 1993a, 1993b). El objetivo, entonces, es relacionar las técnicas que pueden estar implicadas en la manufactura de los artefactos líticos y las maneras en que puede transmitirse esa información.

Se plantean dos escalas de trabajo. Desde una perspectiva regional (escala macro) se busca abordar el abastecimiento de materia prima lítica, no sólo considerando la disponibilidad, calidad y distancia a las fuentes, sino también evaluando la posibilidad del traslado de materias primas como indicador de redes sociales (Gamble 1993a, 1993b; Soffer 1991) que podrían estar enfatizando, entre otros aspectos, el intercambio de información. Desde este punto de vista, ciertos elementos del diseño de los artefactos tallados pueden dotarnos de datos que nos permitan pensar en el flujo de información entre diferentes zonas de la Patagonia centro-meridional. Las áreas que aquí se analizan son, por un lado, el noroeste de la provincia de Santa Cruz, particularmente el área norte del Lago Pueyrredón-Cochrane y, por el otro, el suroeste de la provincia de Chubut, área de El Chalía y Casa de Piedra (Aldea Beleiro), todas ellas ubicadas en la República Argentina.

La micro-escala (Dobres 1999; Torrence 2001) se aborda a partir de la identificación de lo que Gamble (1993a, 1993b) denomina redes «efectivas» o «íntimas», ya señaladas, analizando la circulación de la información en el ámbito del sitio a través de la transmisión del conocimiento de la talla lítica, ya que, como se ha mencionado, estas redes enfatizan la «interacción cara a cara» entre los participantes. La caracterización de diferentes modos de hacer, junto con el análisis de la distribución de actividades en los sitios, podría ayudarnos a entender esas formas de interacción entre los individuos.

Este trabajo se encuentra integrado en dos proyectos mayores que tienen entre sus objetivos estudiar la vinculación entre la movilidad y la disponibilidad de recursos y, por otro lado, discutir el manejo de recursos y las interacciones entre las sociedades cazadoras-recolectoras del sector nordeste de la cuenca del Lago Pueyrredón-Cochrane (véase, entre otros, Aschero et al. 2009, 2010; Sacchi 2012) y el suroeste de la provincia de Chubut (véase, entre otros, Castro 2010; Castro et al. 2011; Pérez de Micou et al. 2008, 2012; Sacchi 2012; Sacchi et al. 2016). A continuación se realiza una breve reseña de las áreas estudiadas (Figura 1).

\subsection{Suroeste de Chubut: Colonia Indigena El Chalia y Aldea Beleiro}

La Colonia Indígena El Chalía se encuentra ubicada en el suroeste de la provincia de Chubut, en la Patagonia argentina. A nivel arqueológico no se contaba con otras investigaciones en la zona que las presentadas por Castro (2010) y Pérez de Micou et al. (2008 y 2012). Entre los trabajos realizados es de importancia el realizado por Castro (2010), ya que subraya la presencia de obsidiana procedente de la fuente de Pampa del Asador, ubicada en la provincia de Santa Cruz, y presenta el primer fechado obtenido para la zona de $c a .470$ años A.P, realizado sobre un tiesto cerámico 
obtenido del sitio Quil 5 en la Laguna Quilchamal, colonia El Chalía. En relación con el área de Aldea Beleiro, se destaca el trabajo de Pérez de Micou y su equipo (Pérez de Micou et al. 2012) en el sitio Casa de Piedra (ubicado en la Estancia Mercedes), una cueva que presenta evidencias arqueológicas en estratigrafía.

Hacia el oeste de las áreas anteriores, en la región de Aisén en Chile, se ubican una serie de sitios que presentan una mayor amplitud temporal en las ocupaciones humanas. El sitio Baño Nuevo 1 se ubica a unos $25 \mathrm{~km}$ de Casa de Piedra (Aldea Beleiro) y a unos 60 km de la Colonia El Chalía; presenta una serie de fechados que abarcan desde los ca. 9500 años A.P hasta los $c a .2800$ años A.P. (Mena et al. 2000).

Castro (2010) ha realizado

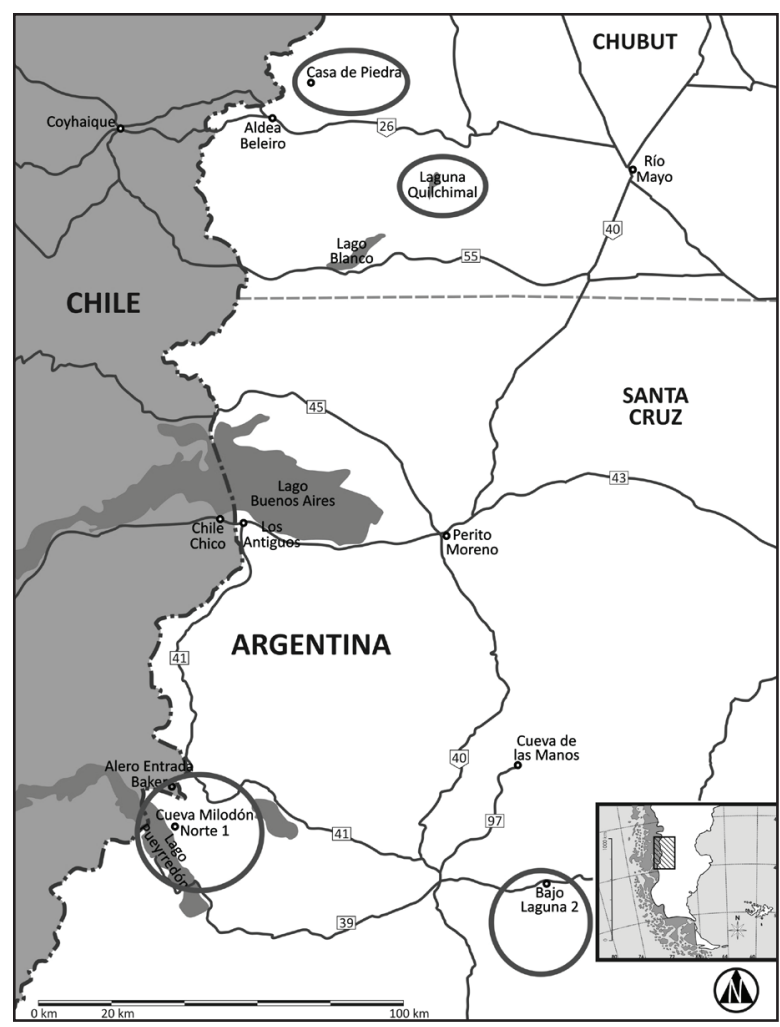

Figura 1: Áreas de estudio varios estudios en la Colonia Indígena El Chalía. Si bien en esta área no se encontraron abrigos o paredones con representaciones rupestres, sí se hallaron una serie de sitios de superficie con abundante material arqueológico. De acuerdo con fuentes históricas y etnográficas, la cuenca del río Senguerr (en la que se encuentra el arroyo Chalía) era utilizada como ruta por parte de las poblaciones indígenas y conectaba el sudoeste de la provincia con la desembocadura del río Chubut donde se encontraba la colonia galesa -actual Trelew-a fines del siglo XIX.

\subsection{Costa nordeste del Lago Pueyrredón-Cochrane}

Las investigaciones sistemáticas en esta área se iniciaron en el sector central de la cuenca, en el sitio Cerro de los Indios 1 -CI1- (Aschero et al. 1999). Las excavaciones en este sitio aportaron las primeras referencias sobre la antigüedad de la ocupación en este sector. Aschero et al. (1999) dividen las ocupaciones, a partir de la secuencia estratigráfica obtenida en CI1, en dos bloques temporales que englobarían -al menos- cinco episodios de ocupación con evidencia de actividades domésticas y procesamiento intensivo de recursos. El bloque inicial está ubicado entre $3860 \pm 90$ 
y $3150 \pm 90$ años AP mientras que el más reciente está comprendido entre los $1810 \pm$ 50 y $990 \pm 110$ años AP. Ambos están separados por un «hiato ocupacional» que fue interpretado como un período de abandono del sitio (De Nigris et al. 2004; Mengoni y Yacobaccio 2000; Yacobaccio y Guráieb 1994). Por otro lado, en la parte baja de la cuenca (el área del lago Salitroso) los fechados más tempranos obtenidos hasta el momento no superan los ca. 2600 años AP (véase, entre otros, Goñi 2000-02, 2011; Goñi y Barrientos 2004; Goñi et al. 2000-02). Sin embargo, en la zona alta de la cuenca, los fechados obtenidos en los sitios Cueva del Milodón Norte 1, Estancia Pueyrredón 1 y 2 y Cerro Cuadrado 3, aumentan la profundidad temporal del poblamiento de la zona hasta $c a .8000$ años AP (véase, entre otros, Aschero et al. 2010; Bozzuto et al. 2012, 2013).

A partir de los fechados obtenidos para los sitios ocupados durante el Holoceno tardío se planteó un modelo de ocupación de la cuenca lacustre (Goñi 2000-02). Dicho modelo propone que la cuenca lacustre baja habría sido utilizada de manera permanente y semi-permanente de forma residencial por parte de los grupos cazadores-recolectores (Goñi 2000-02, 2011). Éste se apoya en la existencia de cambios climáticos y ecológicos durante el Holoceno tardío (Goñi 2000-02; Goñi et al. 2000-02; Goñi y Barrientos 2004) que consistirían en un proceso de progresiva (aunque fluctuante) desecación ambiental que habría comenzado ca. 2500/2000 años AP (Stine y Stine 1990) e intensificado hacia ca. 900 años AP con el desarrollo del fenómeno conocido como Anomalía Climática Medieval (Stine 1994, 2000). Estas condiciones de aridez habrían provocado un cambio en la estructura de recursos y habrían afectado, principalmente, a la existencia y tamaño de los cuerpos de agua (Goñi 2011). Estas nuevas características ecológicas resaltan la ampliación de una estepa arbustiva caracterizada por la presencia de molle (Schinus polygamus) y el descenso de los niveles de diferentes cuencas de la región, principalmente lacustres (Goñi 2011). De esta manera, la disponibilidad de leña y de condiciones ambientales menos hostiles (teniendo en cuenta el carácter marcadamente estacional de Patagonia) se vuelven fundamentales. En tal situación, las cuencas lacustres que presentasen la disponibilidad de este tipo de recursos y condiciones se habrían convertido en espacios privilegiados para la ocupación humana. En los momentos de sequía se habría dado - de acuerdo con lo que plantean Goñi et al. (2000-02)un proceso de concentración de la población en los sectores bajos de la cuenca, que tendrían ocupaciones de tipo residencial más duraderas; los sectores más altos, como las mesetas que circundan la cuenca de los lagos Pueyrredón-PosadasSalitroso, habrían sido visitados solamente de forma logística. Por otro lado, en la costa nordeste del lago Pueyrredón Aschero et al. (2009) propusieron que, dado que los sitios con fechados de $c a .5000$ años AP se ubicaban por encima de la cota de $300 \mathrm{~m}$, era posible hallar en estos niveles o en cotas superiores sitios con fechados similares o más antiguos -como por ejemplo los obtenidos en el sitio Alero Entrada Baker de ca. 7500 años AP, ubicado en las nacientes del río Chacabuco en Chile-.

Hacia el norte, los trabajos desarrollados en la zona de Los Antiguos-Monte Zeballos y Paso Roballos registraron ocupaciones cuyas dataciones están comprendidas entre los ca. 900 y 2100 años AP (Mengoni et al. 2009). Se observa un contraste entre las ocupaciones del sector norte de esta área (Los Antiguos-Monte Zeballos) y 
las del sector sur (Paso Roballos). Siguiendo estas evidencias, los autores plantean una mayor continuidad en el uso de los lugares en el sector sur, caracterizado por la reocupación de espacios previamente utilizados, al igual que por ocupaciones más prolongadas que las observadas en el sector norte (Mengoni et al. 2009).

\section{Planteamiento del problema: acerca de la circulación de información, la transmisión del conocimiento y la tecnología lítica}

Como se ha adelantado en la sección anterior, tomamos la movilidad de los grupos como algo que se encuentra inserto en el establecimiento y mantenimiento de redes sociales a través de las cuales fluye no sólo información, sino también gente y materias primas. Consideramos que estas redes son la combinación de diversos tipos de movimientos, sean estos individuales o familiares (Whallon 2006), y que los artefactos intervienen en ellas. Aquí los individuos ejercen la negociación, afiliación y reciprocidad. Las redes sociales, entonces, afectan a decisiones en todos los aspectos de la vida de un individuo (Gamble 1993a, 1993b). Un mismo individuo interactúa en redes que funcionan a escalas diferentes. Resultan de interés para este trabajo las redes íntima, efectiva y extendida o ampliada (en el sentido utilizado por Gamble 1993a, 1993b, 1999). Las dos primeras corresponderían a las personas que proveen al individuo de la asistencia material y emocional durante la vida diaria. La red efectiva supone un tamaño mayor que la íntima, pero es menos permanente y estable que ésta última (Gamble 1993a, 1993b). A medida que las redes expanden su tamaño, los lazos que unen a los individuos que las integran se vuelven menos estrechos.

Para poder abarcar estas redes desde el punto de vista arqueológico, es necesario trabajar en diferentes escalas. La micro-escala (el análisis de los conjuntos a nivel de sitio) se aborda en este trabajo desde el análisis de la variabilidad específica dentro de los conjuntos (Dobres 1999). El sitio, entonces, es el ámbito en el cual los agentes prehistóricos interactuaron tanto material como socialmente. Tomando todas las salvedades del caso y teniendo como principal supuesto que el registro arqueológico es un registro promediado -la suma de los restos materiales de conductas practicadas en diversos momentos temporales-(Borrero 2001), consideramos que es posible generar puentes entre la micro-escala y las redes que estarían operando a una escala mayor -la red extendida- buscado las posibles relaciones entre las distintas variabilidades producto de las conductas humanas.

Una parte de la información que podría transmitirse a través del material lítico y que sería factible recuperar arqueológicamente (trabajando en la pequeña escala, como se plantea en esta investigación) serían los episodios de aprendizaje de talla lítica. Como se ha argumentado en diversas publicaciones (Bodu et al. 1990; Karlin y Julien 1994; Finlay 1997; Hocsman 2006; Sacchi 2009a, 2009b, 2010, entre otros), el proceso de reducción de un instrumento implica un saber cómo (know how) realizar esas actividades. Ahora bien, este saber cómo debe ser transmitido en algún momento para que no se pierda dentro de la trayectoria de un grupo. Aprendizaje y conocimiento, entonces, implican relaciones entre los individuos y su contexto social (Lave y 
Wenger 1991, en Grimm 2000). La adquisición de habilidades técnicas es un proceso complejo que implica el aprender cómo actuar para resolver los problemas que se plantean durante la talla, más que una cuestión relacionada únicamente con fórmulas motoras (Grimm 2000; Stout 2002). El aprendizaje ocurre, de esta forma, a través de la relación de los aprendices con la práctica de los expertos. Y es a través de ella que se genera, también, un sentimiento de comunidad. Podríamos pensar estas evidencias de aprendizaje, de distintas formas de tallar, como indicadores de las redes sociales más pequeñas, esto es: la red íntima y la efectiva.

Debemos tener en cuenta que el uso del espacio y la movilidad de los grupos influyeron en las diferentes estrategias que éstos implementaron para aprovisionarse de materias primas. Así, no esperaríamos encontrar materias primas de alta calidad trabajadas por talladores inexpertos, sino que éstos utilizarían materiales de calidad mediana a baja. Por ejemplo, en eventos de movilidad residencial muy alta, como serían los momentos de exploración y/o colonización inicial de un espacio (sería el caso de las primeras ocupaciones del sitio Cueva del Milodón Norte 1 -ubicado en la costa norte del lago Pueyrredón, en la provincia de Santa Cruz-), no esperaríamos encontrar rastros de talladores inexpertos, cosa que sí sucedería, al menos hipotéticamente, durante las ocupaciones que respondieran a momentos de ocupación efectiva del espacio.

De acuerdo con el problema de investigación planteado, se decidió tomar como bloque temporal el Holoceno medio y tardío, ya que, según la información disponible para las áreas estudiadas, podría corresponder a una ocupación efectiva del espacio (Borrero 1994-95). Es en esos periodos cuando podría estar ocurriendo lo que Kuhn (2004) denomina aprovisionamiento de lugares, o bien una mixtura entre aprovisionamiento de individuos y de lugares. Si tomamos este modelo y lo agregamos a las categorías de red planteadas más arriba, podríamos pensar en individuos equipados que viajan para «visitar» a otros grupos y, en esa visita, intercambian o adquieren instrumentos o materias primas; en esos mismos viajes los menos expertos «aprenden» nuevas técnicas.

En el caso de la costa noreste del lago Pueyrredón-Cochrane, el material que aquí se analiza proviene de aleros o cuevas cuyos fechados más antiguos, por el momento, son de $c a .7800$ años A.P (Aschero et al. 2009, 2010; Bozzuto et al. 2012, 2013) y cuya secuencia de ocupaciones llega hasta el Holoceno tardío. Teniendo en cuenta la redundancia ocupacional que se menciona para las áreas del noroeste de Santa Cruz, hacer un análisis en detalle de los desechos de talla y núcleos nos permite obtener evidencias para discutir la presencia de talladores con diferentes habilidades técnicas, lo cual podría tomarse, a su vez, como un posible indicador de lo que se denomina red íntima o efectiva, porque se trataría de la transmisión de información a un nivel de sitio.

Para el bloque temporal correspondiente al Holoceno tardío, en las páginas siguientes se analiza el material proveniente del sitio Bajo de la Laguna 2 (BLG2), que presenta un fechado de $c a$. 169 años A.P (Sacchi 2012, 2013), así como las capas 1 a 8 del sitio Cueva del Milodón Norte 1. En el caso del suroeste de Chubut, se analizan aquí materiales provenientes de recolecciones superficiales, así como el material proveniente del sitio Casa de Piedra fechado en $c a .490$ años AP (Pérez de Micou et al. 2012; Sacchi 2012). 
Asimismo, con este trabajo a escala de sitio podríamos identificar variaciones morfológicas (que podrían responder a diseños compartidos) que nos posibiliten luego comparar los sitios entre sí. Esto nos permitiría unir la escala de sitio con la escala espacial más amplia, evaluando así las similitudes en el registro arqueológico a nivel regional.

\section{Material experimental: red íntima y adquisición de habilidades para la talla}

¿Cómo se puede identificar el aprendizaje de la talla en el material lítico? A partir de los resultados obtenidos en distintos trabajos de arqueología experimental (Sacchi 2009a, 2009b, 2010, 2012, 2013, 2014) y los datos bibliográficos sobre la temática específica de los aprendices (Bodu et al. 1990; Finlay 1997; Karlin y Julien 1994), se han detectado algunas tendencias significativas para su comparación con el material arqueológico.

En el caso de los desechos de talla, destacan las diferencias observadas entre los talladores en la relación ancho/espesor de talones: se ha podido identificar que los talladores con menor experiencia obtienen talones más anchos y espesos (Cuadro 1; véase, entre otros, Bodu et al. 1990; Sacchi 2009b, 2014). Otra diferencia que llama la atención (ver Figura 2) es la presencia de marcas de impacto en los talones, variable que muestra altos porcentajes en el material tallado por inexpertos (Bodu et al. 1990; Sacchi 2009b). Por último, es interesante el resultado obtenido en la comparación de terminaciones, presentando, en el caso del tallador experto, un alto porcentaje de terminaciones agudas, mientras que los talladores menos hábiles tienen una mayor diversidad y un porcentaje más alto de terminaciones en charnela y fracturadas ( $\mathrm{Fi}$ gura 3; Sacchi 2014).

En el caso del análisis de núcleos, como se ha mencionado en publicaciones anteriores (véase, entre otras, Bodu et al. 1990; Karlin y Julien 1994; Sacchi 2009a, 2012, 2014), ciertas características permiten diferenciar el material experimental producto de la talla de expertos e inexpertos. De la comparación entre la cantidad de charnelas observadas y la cantidad de extracciones, cuya proporción se ha denominado índice de eficiencia (Sacchi 2014) ${ }^{1}$, pudo observarse que los talladores con mayor experiencia obtenían valores más cercanos a 0 mientras que los talladores inexpertos se acercaban a 1 (Cuadro 2).

Estos resultados sugieren que los talladores más expertos manejarían de manera más «eficiente» los núcleos y por consiguiente lograrían un mejor tratamiento de las distintas materias primas. Por otro lado, dentro de los materiales experimentales, y como era de esperar, los ángulos de descarte de los núcleos producto de la acción de expertos e inexpertos presentaron resultados diferentes entre sí, pero similares a lo observado en relación con la eficiencia. Las tendencias muestran una propensión, entre los talladores menos hábiles, al abandono de los núcleos cuando las plataformas aún se encuentran activas o parcialmente agotadas (Bodu et al. 1990; Karlin y Julien

1 El índice de eficiencia se obtiene de la razón entre la cantidad de charnelas y la cantidad de extracciones observadas en cada uno de los núcleos analizados. 
Tipos de talón

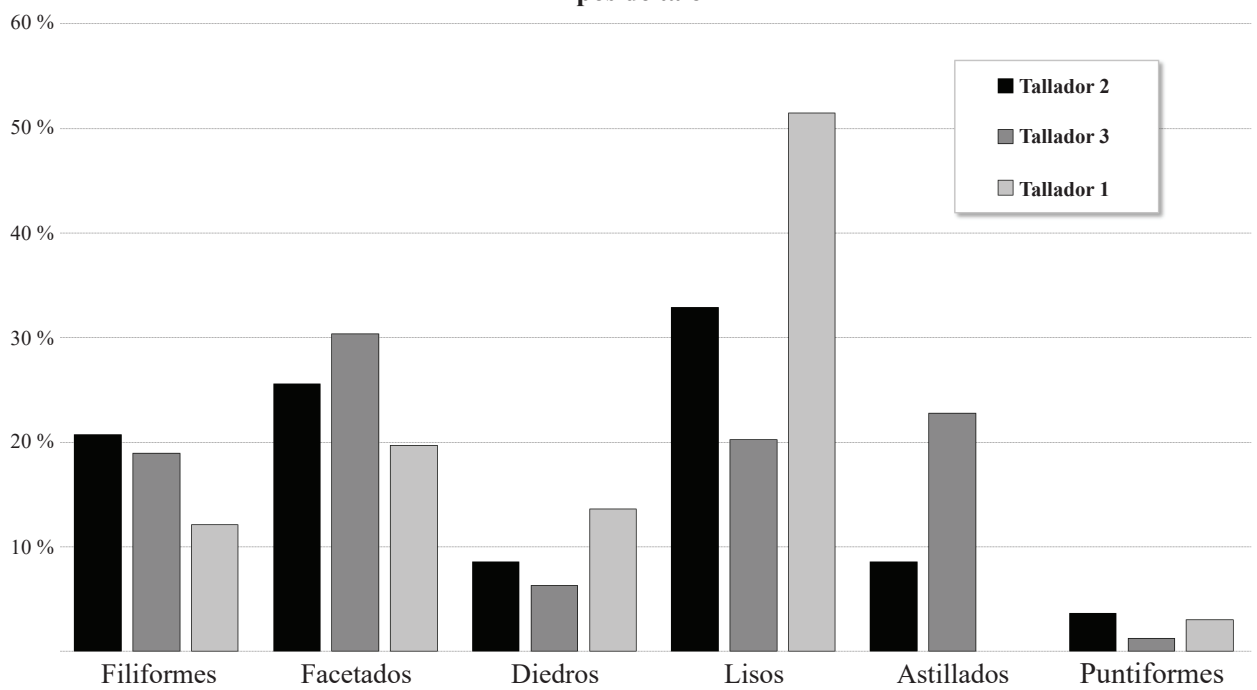

Figura 2: Porcentajes de tipos de talón presentes comparados entre los distintos talladores; el tallador 1 representa al tallador con mayor experiencia, el tallador 3 a un tallador sin experiencia previa y el tallador 2 a un tallador con experiencia media/escasa.

(Tomado de Sacchi 2009b y 2012).

\section{Tipos de terminación}

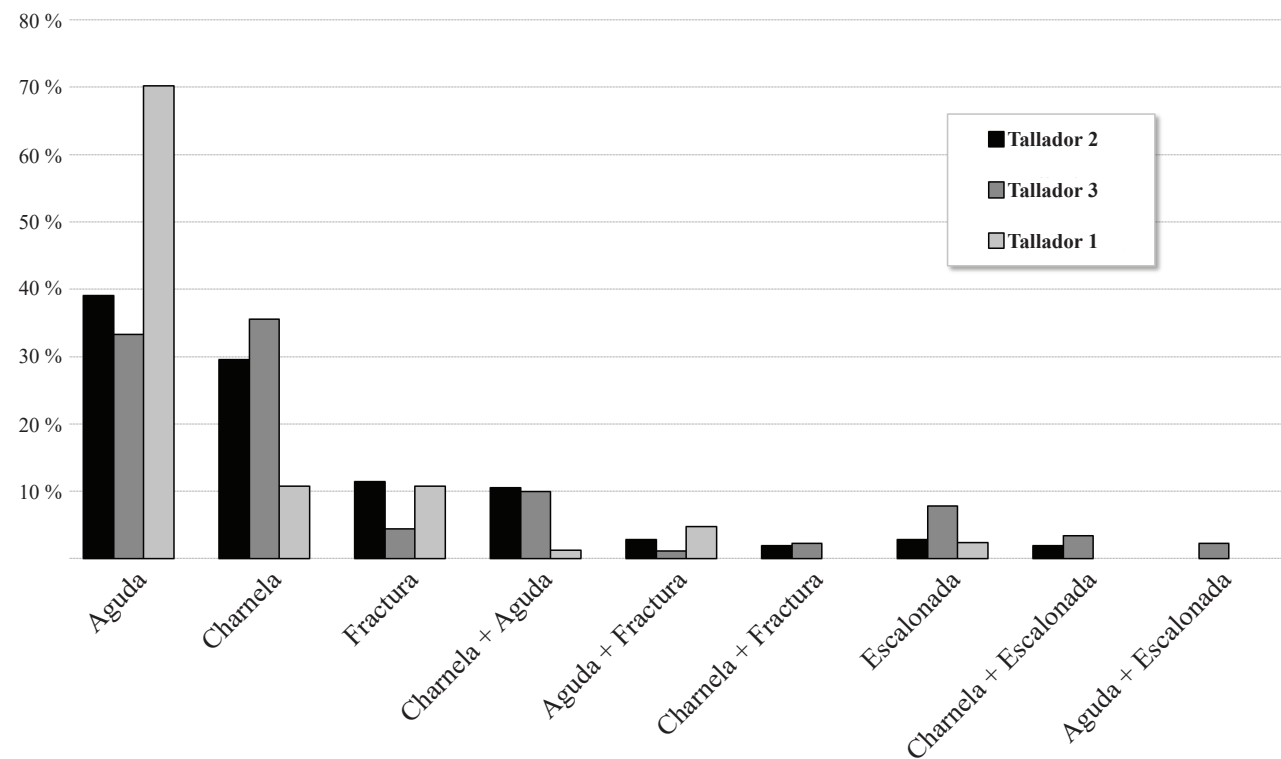

Figura 3: Comparación entre los tipos de terminación presentes en los distintos talladores. ( $N=233$. Tomado de Sacchi 2009b y 2012). 
Cuadro 1: Intervalos de anchura del talón en los productos de tres talladores con diferente grado de experiencia (tomado de Sacchi 2009b)

\begin{tabular}{llllllll}
\hline & \multicolumn{7}{c}{ Intervalos } \\
\cline { 2 - 8 } & $\mathbf{1 - 4}$ & $\mathbf{5 - 8}$ & $\mathbf{9 - 1 2}$ & $\mathbf{1 3 - 1 5}$ & $\mathbf{1 6 - 1 9}$ & $\mathbf{2 0 - 2 3}$ & $>\mathbf{2 3}$ \\
\hline Tallador 3 & 2 & 12 & 16 & 13 & 9 & 8 & 21 \\
& $2,47 \%$ & $14,81 \%$ & $19,75 \%$ & $16,05 \%$ & $11,11 \%$ & $9,88 \%$ & $25,93 \%$ \\
Tallador 2 & 2 & 13 & 15 & 8 & 13 & 5 & 28 \\
& $2,38 \%$ & $15,48 \%$ & $17,86 \%$ & $9,52 \%$ & $15,48 \%$ & $5,95 \%$ & $33,33 \%$ \\
Tallador 1 & 2 & 8 & 17 & 11 & 8 & 11 & 12 \\
& $2,90 \%$ & $11,59 \%$ & $24,64 \%$ & $15,94 \%$ & $11,59 \%$ & $15,94 \%$ & $17,39 \%$ \\
\hline
\end{tabular}

Cuadro 2: Índice de eficiencia de tres talladores con diferente grado de experiencia (tomado de Sacchi 2003a)

\begin{tabular}{lccc}
\hline & Charnelas & Extracciones & Eficiencia \\
\hline Tallador 3 & 6 & 10 & 0,6 \\
Tallador 3 & 7 & 14 & 0,5 \\
Tallador 2 & 4 & 9 & 0,444 \\
Tallador 1 & 1 & 12 & 0,083 \\
Tallador 1 & 1 & 12 & 0,083 \\
\hline
\end{tabular}

1994; McCall 2011; Sacchi 2014), presentando una serie de errores que no podrían solucionar; en los núcleos trabajados por el tallador con mayor experiencia los ángulos de descarte son mayores y las plataformas se encuentran totalmente agotadas (Sacchi 2014: tablas 3 y 4). Características similares pudieron observarse en la muestra experimental de artefactos producidos por talla bifacial. Destaca una cantidad importante de charnelas y melladuras en las aristas, resultados esperables en la talla bifacial por parte de talladores con poca habilidad técnica (Bodu et al. 1990; Finlay 1997; Grimm 2000; McCall 2011; Stout 2002). Por otro lado, en algunos casos se observan características en la cara ventral de las formas base que demostrarían una impericia en el adelgazamiento de los bulbos. Asimismo, la mayoría de los lascados de formatización ${ }^{2}$ no logran superar el centro de la pieza y se encuentran restringidos a los bordes. Los resultados obtenidos por Hocsman (2006) para el análisis de bifaces arqueológicos del área de Antofagasta de la Sierra, en Catamarca, presentan muchas similitudes con el material experimental (Sacchi 2014: figs. 2 y 3): el autor observa en los bordes presencia de machacados, fracturas y sobre-engrosamiento, así como, en las extracciones realizadas en la cara de los bifaces, terminaciones en charnela y quebradas y negativos de lascado «estallados», sumados a la falta de simetría en el plano longitudinal y a las aristas sinuosas irregulares (Finlay 1997; Grimm 2000; Hocsman 2006; Stout 2002; Whittaker 1994).

En publicaciones anteriores (Sacchi 2009a, 2009b, 2010, 2014, entre otras) se discutió sobre la utilización del término «aprendices» o de la expresión «talladores inexpertos» en su lugar. Se decidió el uso de ésta última, ya que remite a diferentes grados de experiencia en la práctica de la talla lítica y permite ampliar la categoría, pudiendo abarcar grupos mayores en los que cabe incluir tanto a «niños» como a adolescentes

\footnotetext{
${ }^{2}$ Se entiende por formatización la modificación de un núcleo, una lasca o una hoja a partir de la talla, sea por percusión o presión, para confeccionar una herramienta. Un objeto lítico formatizado es, por tanto, aquél cuyas caras o bordes han sido modificados por percusión o presión.
} 
y a adultos, dependiendo del grado de experiencia que posean. No obstante, también debe tenerse en cuenta que el grado de habilidad para la talla puede perderse; sería el caso de grandes talladores que, debido a enfermedades u otros problemas, han visto reducidas sus habilidades motoras y, por lo tanto, su habilidad para la talla ha disminuido. Por ello, en diversas publicaciones se ha optado por hablar de inexpertos y de diferentes grados de pericia técnica (Galarce 2008; Hocsman 2006; Sacchi 2009a, 2009b, 2014, entre otros). De acuerdo con las habilidades técnicas, una de las diferencias esperable, en el caso de los desechos de talla, es la de encontrar evidencias de fuerza excesiva en los golpes, como por ejemplo bulbos de percusión muy espesos y marcados, que pueden deberse al uso de un percutor inadecuado o a la ejecución de golpes muy fuertes (Whittaker 1994). Otra característica esperable en los trabajos de talladores con poca experiencia es la inclinación de la plataforma de percusión: se esperarían plataformas muy oblicuas cuando el ángulo elegido para el golpe no es el más adecuado, Ambos rasgos, sumados al espesor de los talones, podrían estar evidenciando poca experiencia en la práctica de la talla (Bodu et al. 1990).

\section{Del material experimental al material arqueológico: resultados y discusión}

Durante las experimentaciones, los talladores con menor experiencia mostraron una mayor cantidad de errores de talla que el experto. Sin embargo, entre los menos hábiles surgieron distinciones que permitieron diferenciarlos entre sí. En el caso de los núcleos analizados de los conjuntos arqueológicos, para el índice de eficiencia, pudieron observarse, en algunos casos, medidas similares o mayores a las de los talladores menos hábiles (Cuadros 3 y 4). Para el análisis del material, tanto arqueológico como experimental, se utilizó la propuesta de Aschero $(1975,1983)$.

En los Cuadros 3 y 4 se presentan los valores de eficiencia para el material arqueológico recuperado en estratigrafía y superficie. Como puede observarse, los sitios Cueva del Milodón Norte 1 (CMN1) y Casa de Piedra 1 (CP1) presentan núcleos con valores cercanos a los de los talladores menos hábiles, mientras que Bajo de la Laguna 2 (BLG2) presenta valores que se acercan mucho más a 0. Esto se vuelve interesante al agregar a esta información los resultados obtenidos de los desechos de talla. En el caso de CP1, a partir del análisis de desechos de talla se pudo observar que la obsidiana, aunque en bajas proporciones, se encuentra en toda la secuencia estratigráfica analizada. Los tamaños que predominan en esta materia prima son, por lo general, muy pequeños lo que nos permitiría inferir tareas de mantenimiento de artefactos y/o producción final (Ericson 1984). En el caso de los núcleos y artefactos formatizados, esta materia prima no se encuentra o se encuentra en proporciones muy bajas (en el caso de los artefactos formatizados aparece únicamente en la capa 4 y en un único espécimen de la recolección de superficie).

Del análisis del material lítico de CP1 se desprende que la capa 4 es la más abundante en cuanto a evidencias recuperadas (Cuadro 5).

En todas las capas se pudieron observar marcas de impacto en los talones, y si bien predominan los espesores delgados y medianamente delgados, al cruzar esta variable con el ancho, las materias primas locales presentan características que, en muchos 
Cuadro 3: Medidas de eficiencia del material en estratigrafía

(en negrita se destacan los valores más altos y cercanos a 1)

\begin{tabular}{llll}
\hline \multicolumn{1}{c}{ Sitio } & \multicolumn{1}{c}{ Origen } & \multicolumn{1}{c}{ Materia prima } & Eficiencia \\
\hline Bajo de la Laguna 2 & Rec. superficie & Obsidiana & 0,29 \\
Bajo de la Laguna 2 & Rec. superficie & Obsidiana & 0,06 \\
Bajo de la Laguna 2 & Rec. superficie & Sílice & 0,4 \\
Bajo de la Laguna 2 & $2(2)$ & Ś́lice & 0 \\
Bajo de la Laguna 2 & $2(2)$ & Obsidiana & 0,12 \\
Bajo de la Laguna 2 & 4 & Obsidiana & 0,2 \\
Cueva del Milodón Norte 1 & 1 & Sílice & 0,25 \\
Cueva del Milodón Norte 1 & 1 & Ślice & 0 \\
Cueva del Milodón Norte 1 & 2 & Riolita silicificada & 0,25 \\
Cueva del Milodón Norte 1 & $\mathbf{2}$ & Sílice & $\mathbf{0 , 5}$ \\
Cueva del Milodón Norte 1 & 6 & Xilópalo & 0,17 \\
Casa de Piedra 1 & $\mathbf{0}$ & Sílice & $\mathbf{0 , 5}$ \\
Casa de Piedra 1 & 0 & Sílice & 0,25 \\
Casa de Piedra 1 & $1(1)$ & Riolita & 0,14 \\
Casa de Piedra 1 & $1(2)$ & Ś́lice & 0,33 \\
Casa de Piedra 1 & $1(3)$ & Sílice & 0 \\
Casa de Piedra 1 & $\mathbf{2}$ & Sílice & $\mathbf{0 , 4}$ \\
\hline
\end{tabular}

Cuadro 4: Medidas de eficiencia del material de superficie (en negrita se destacan los valores cercanos o mayores a los obtenidos en el material experimental)

\begin{tabular}{|c|c|c|c|c|c|}
\hline Sitio & $\begin{array}{c}\text { Materia } \\
\text { prima }\end{array}$ & Eficiencia & Sitio & $\begin{array}{c}\text { Materia } \\
\text { prima }\end{array}$ & Eficiencia \\
\hline Laguna Quilchamal & Sílice & 0,63 & Tapera Almeida & Sílice & 0,67 \\
\hline Laguna Quilchamal & Sílice & 0,67 & Tapera Almeida & Sílice & 0,25 \\
\hline Laguna Quilchamal & Sílice & 0,1 & Tapera Almeida & Sílice & 0,33 \\
\hline Laguna Quilchamal & Sílice & 0,42 & Tapera Almeida & Sílice & 0,2 \\
\hline Laguna Quilchamal & Sílice & 0 & Tapera Almeida & Sílice & 0,2 \\
\hline Laguna Quilchamal & Basalto & 0,2 & Tapera Almeida & Sílice & 0,8 \\
\hline Laguna Quilchamal & Basalto & 0 & Tapera Almeida & Sílice & 0,25 \\
\hline Laguna Quilchamal & Dacita & 0,29 & Tapera Almeida & Sílice & 0,4 \\
\hline Laguna Quilchamal & Xilópalo & 0,43 & Tapera Almeida & Sílice & 0,1 \\
\hline Laguna Quilchamal & Obsidiana & 0 & Tapera Almeida & Sílice & 0 \\
\hline Tapera Almeida & Obsidiana & 0,1 & Tapera Almeida & Sílice & 0,17 \\
\hline Tapera Almeida & Obsidiana & 0,2 & Tapera Almeida & Sílice & 0 \\
\hline Tapera Almeida & Basalto & 0,4 & Tapera Almeida & Sílice & 0 \\
\hline Tapera Almeida & Basalto & 0,67 & Tapera Almeida & Sílice & 0,33 \\
\hline Tapera Almeida & Basalto & 0,3 & Tapera Almeida & Riolita & 0,29 \\
\hline Tapera Almeida & Basalto & 0,45 & Tapera Almeida & Riolita & 0 \\
\hline Tapera Almeida & Basalto & 0,27 & Tapera Almeida & Riolita & 0 \\
\hline Tapera Almeida & Basalto & 0,7 & Tapera Almeida & Calcedonia & 0,14 \\
\hline Tapera Almeida & Basalto & 0 & Tapera Almeida & Cuarcita & 0,38 \\
\hline Tapera Almeida & Basalto & 0,13 & & & \\
\hline
\end{tabular}

casos, son similares a las observadas en el material experimental. En líneas generales las rocas silíceas (consideradas de acceso local) presentan con mayor recurrencia terminaciones en charnela y bulbos dobles, así como anchos mayores y espesores medianos en los talones. Esto es totalmente diferente a lo que sucede con la obsidiana, que presenta en todos los casos los menores tamaños. Debemos destacar que llama la 
Cuadro 5: Estructura de la muestra dividida por clase tipológica

(según Aschero 1975 y 1983) y capa de procedencia $(\mathrm{N}=947)$

\begin{tabular}{|c|c|c|c|c|c|c|c|c|c|}
\hline Clase tipológica & C.0 & C.1 (1) & C.1 (2) & C.1 (3) & C. 2 & C.3 & C.4 (1) & C.4 (2) & Total \\
\hline Desechos & 44 & 40 & 42 & 104 & 126 & 95 & 303 & 145 & 866 \\
\hline $\begin{array}{l}\text { Artefactos for- } \\
\text { matizados }\end{array}$ & 4 & 2 & 1 & 0 & 2 & 2 & 19 & 2 & 32 \\
\hline $\begin{array}{l}\text { Filos naturales } \\
\text { con rastros com- } \\
\text { plementarios }\end{array}$ & 2 & 0 & 1 & 0 & 0 & 2 & 5 & 0 & 10 \\
\hline Núcleos & 2 & 1 & 1 & 1 & 1 & 0 & 0 & 0 & 6 \\
\hline Total & 54 & 43 & 45 & 105 & 129 & 99 & 327 & 147 & 947 \\
\hline
\end{tabular}

atención la presencia de talones astillados en calcedonia principalmente; esto podría indicar evidencias de talla bipolar, o machacado de los talones por golpes excesivos, como pudo observarse en el material experimental (Cuadro 6).

En el caso de CMN1, las rocas silíceas, en conjunto con la riolita silicificada, presentan, en cuanto a desechos de talla, evidencias de bulbos dobles, marcas de impacto, terminaciones en charnela y anchos de talones mayores (Cuadro 9).

Sin embargo, necesitaría ampliarse la muestra de análisis para generar una base de datos mayor, ya que las muestras analizadas se encuentran muy fragmentadas. Más allá de esto, pudieron observarse ciertas tendencias en cuanto a las materias primas presentes: predominan en orden de importancia las rocas silíceas, seguidas por la obsidiana y el basalto (aunque en proporciones bajas). En cuanto a los desechos de talla, pudo observarse la recurrencia de ciertas características que, comparando con los resultados del material experimental, podrían asociarse a talladores con menor habilidad en la talla, a saber: la presencia de talones astillados, terminaciones en charnela y marcas de impacto, talones cuyos anchos se agrupan entre 13 y 23 milímetros.

Se destaca también que, a lo largo del análisis de desechos, muchas de estas características se observaron en obsidiana, cosa que llama la atención. Sin embargo, los mayores porcentajes y recurrencias de este tipo de características se observaron en las rocas silíceas, que podrían abastecerse en las cercanías del sitio. Las capas más tempranas analizadas para este sitio (capas 7 y 8 ) presentan ciertas características que llaman la atención, como la preponderancia de marcas de impacto y terminaciones en charnela en obsidiana y basalto, características que se combinan con talones relativamente delgados.

A este respecto, Reynolds (2011), retomando la propuesta de Stout (2002) en su comparación de conjuntos experimentales y arqueológicos, plantea que, si bien las diferencias entre talladores hábiles y menos hábiles pueden darse en cuestiones de tamaño (los talladores expertos obtienen por lo general lascas más largas y delgadas; véase Sacchi 2009b, 2010), debemos asumir que los tamaños de las lascas obtenidas dependen de distintos factores como, por ejemplo, entre otros, la conservación de las materias primas, el tipo de instrumentos que se quiere confeccionar, el tamaño del núcleo y la variabilidad entre talladores. En este caso volvemos a BLG2, sitio en el que la aparición de errores de talla (talones más anchos, terminaciones en charnela, presencia de talones astillados, y núcleos abandonados prematuramente) presenta bajos porcentajes que, sumados al tipo de artefactos observados nos hacen pensar en un 
Cuadro 6: Tipos de talón presentes en los desechos de Casa de Piedra 1 (Chubut)

\begin{tabular}{lcrrrrrr}
\hline & Cortical & \multicolumn{1}{c}{ Liso } & Punti. & Facetado & Diedro & Astillado & Filiforme \\
\hline Obsidiana & $0,00 \%$ & $0,76 \%$ & $0,25 \%$ & $1,27 \%$ & $0,25 \%$ & $0,00 \%$ & $3,50 \%$ \\
Basalto & $0,25 \%$ & $1,77 \%$ & $0,00 \%$ & $0,25 \%$ & $1,01 \%$ & $0,00 \%$ & $1,20 \%$ \\
Sílice & $2,28 \%$ & $23,29 \%$ & $0,76 \%$ & $9,11 \%$ & $2,78 \%$ & $1,70 \%$ & $6,80 \%$ \\
Otras & $0,25 \%$ & $3,04 \%$ & $0,00 \%$ & $2,53 \%$ & $1,52 \%$ & $0,50 \%$ & $1,50 \%$ \\
Toba silicificada & $0,51 \%$ & $9,37 \%$ & $0,25 \%$ & $1,77 \%$ & $0,76 \%$ & $0,50 \%$ & $1,00 \%$ \\
Riolita silicificada & $0,51 \%$ & $5,06 \%$ & $0,00 \%$ & $2,28 \%$ & $1,27 \%$ & $0,50 \%$ & $2,30 \%$ \\
Riolita & $0,25 \%$ & $3,80 \%$ & $0,00 \%$ & $2,28 \%$ & $0,76 \%$ & $0,00 \%$ & $0,00 \%$ \\
\hline
\end{tabular}

Cuadro 7: Intervalos de ancho de talón de Casa de Piedra 1 (Chubut)

\begin{tabular}{lrrrcccc}
\hline & \multicolumn{7}{c}{ Intervalos } \\
\cline { 2 - 8 } & $\mathbf{1 - 4}$ & $\mathbf{5 - 8}$ & $\mathbf{9 - 1 2}$ & $\mathbf{1 3 - 1 5}$ & $\mathbf{1 6 - 1 9}$ & $\mathbf{2 0 - 2 3}$ & $>\mathbf{2 3}$ \\
\hline Obsidiana & $2 \%$ & $3 \%$ & $1 \%$ & $0 \%$ & $0 \%$ & $0 \%$ & $0 \%$ \\
Basalto & $0 \%$ & $2 \%$ & $1 \%$ & $1 \%$ & $0 \%$ & $1 \%$ & $0 \%$ \\
Sílice & $11 \%$ & $18 \%$ & $8 \%$ & $4 \%$ & $3 \%$ & $2 \%$ & $2 \%$ \\
Otras & $1 \%$ & $4 \%$ & $1 \%$ & $1 \%$ & $1 \%$ & $0 \%$ & $1 \%$ \\
Toba silicificada & $1 \%$ & $6 \%$ & $4 \%$ & $1 \%$ & $2 \%$ & $0 \%$ & $0 \%$ \\
Riolita silicificada & $3 \%$ & $3 \%$ & $3 \%$ & $1 \%$ & $1 \%$ & $1 \%$ & $1 \%$ \\
Riolita & $1 \%$ & $3 \%$ & $2 \%$ & $1 \%$ & $2 \%$ & $1 \%$ & $1 \%$ \\
Total & $18 \%$ & $37 \%$ & $21 \%$ & $7 \%$ & $9 \%$ & $5 \%$ & $4 \%$ \\
\hline
\end{tabular}

Cuadro 8: Porcentajes de tipos de terminación en desechos de talla de Casa de Piedra 1 (Chubut)

\begin{tabular}{lrccc}
\hline & Aguda & Quebrada & Fracturada & Charnela \\
\hline Obsidiana & $3 \%$ & $0 \%$ & $3 \%$ & $1 \%$ \\
Basalto & $3 \%$ & $0 \%$ & $1 \%$ & $0 \%$ \\
Sílice & $27 \%$ & $1 \%$ & $14 \%$ & $4 \%$ \\
Otras & $4 \%$ & $0 \%$ & $4 \%$ & $1 \%$ \\
Toba silicificada & $6 \%$ & $1 \%$ & $6 \%$ & $1 \%$ \\
Riolita silicificada & $5 \%$ & $0 \%$ & $5 \%$ & $2 \%$ \\
Riolita & $5 \%$ & $0 \%$ & $2 \%$ & $1 \%$ \\
Total & $54 \%$ & $1 \%$ & $35 \%$ & $10 \%$ \\
\hline
\end{tabular}

espacio de actividades limitadas. En un sitio de estas características no se esperaría obtener evidencias de talladores menos hábiles ya que se realizan tareas demasiado específicas (Cuadros 12 y 13).

En el caso de las concentraciones analizadas, todas ellas del SO de Chubut, en el Cuadro 4 se presentaron los valores de eficiencia obtenidos de los núcleos. Resaltados pueden observarse los valores cercanos o mayores a los obtenidos en el material experimental. Se destaca que la recurrencia de charnelas por cantidad de extracciones es mayor en las rocas definidas como inmediatamente disponibles, como pueden ser las rocas silíceas y el basalto (Castro 2010), es decir tenían un uso menos eficiente. En cambio, la obsidiana, de procedencia lejana, se encuentra aprovechada hasta el agotamiento de los núcleos, que en su mayoría fueron terminados por la técnica bipolar.

Ahora bien, en el caso de los desechos de talla, en Quil 5 se observó una mayor representación de tipos de talón, a diferencia de lo que se pudo observar en Tapera Almeida-TA- (Sacchi 2010: figs. 1, 2, 4 y 5). En este sitio predominan los talones lisos y facetados. En primera instancia esta variable fue cruzada con los tipos de ter- 
Cuadro 9: Intervalos de ancho de talón de Cueva del Milodón Norte 1 (Santa Cruz)

\begin{tabular}{lrrrrrrr}
\hline \multicolumn{1}{c}{ Materia prima } & \multicolumn{7}{c}{ Intervalos } \\
\cline { 2 - 8 } & $\mathbf{1 - 4}$ & $\mathbf{5 - 8}$ & $\mathbf{9 - 1 2}$ & $\mathbf{1 3 - 1 5}$ & $\mathbf{1 6 - 1 9}$ & $\mathbf{2 0 - 2 3}$ & $>\mathbf{2 3}$ \\
\hline Obsidiana & $16 \%$ & $12 \%$ & $3 \%$ & $1 \%$ & $1 \%$ & $0 \%$ & $0 \%$ \\
Basalto / And. & $2 \%$ & $4 \%$ & $2 \%$ & $2 \%$ & $0 \%$ & $0 \%$ & $1 \%$ \\
Silice & $16 \%$ & $14 \%$ & $0 \%$ & $0 \%$ & $0 \%$ & $0 \%$ & $0 \%$ \\
Calcita & $3 \%$ & $4 \%$ & $1 \%$ & $0 \%$ & $0 \%$ & $0 \%$ & $0 \%$ \\
Limolita & $0 \%$ & $1 \%$ & $1 \%$ & $0 \%$ & $0 \%$ & $0 \%$ & $0 \%$ \\
Riolita silicificada & $3 \%$ & $3 \%$ & $0 \%$ & $0 \%$ & $0 \%$ & $0 \%$ & $0 \%$ \\
Rta & $0 \%$ & $0 \%$ & $0 \%$ & $0 \%$ & $0 \%$ & $0 \%$ & $0 \%$ \\
Toba & $1 \%$ & $0 \%$ & $0 \%$ & $0 \%$ & $0 \%$ & $0 \%$ & $0 \%$ \\
Toba silicificada & $1 \%$ & $3 \%$ & $0 \%$ & $0 \%$ & $0 \%$ & $0 \%$ & $0 \%$ \\
Cuarcita & $0 \%$ & $0 \%$ & $0 \%$ & $0 \%$ & $0 \%$ & $0 \%$ & $0 \%$ \\
Xilópalo & $0 \%$ & $1 \%$ & $0 \%$ & $0 \%$ & $0 \%$ & $0 \%$ & $0 \%$ \\
Total & $43 \%$ & $42 \%$ & $8 \%$ & $3 \%$ & $2 \%$ & $1 \%$ & $1 \%$ \\
\hline
\end{tabular}

Cuadro 10: Porcentajes de tipo de terminación en desechos de talla de Cueva del Milodón Norte 1 (Santa Cruz)

\begin{tabular}{lrccc}
\hline \multicolumn{1}{c}{ Materia prima } & Aguda & Charnela & Quebrada & Fractura \\
\hline Obsidiana & $17 \%$ & $4 \%$ & $1 \%$ & $11 \%$ \\
Basalto / And. & $5 \%$ & $1 \%$ & $0 \%$ & $5 \%$ \\
Silice & $18 \%$ & $1 \%$ & $0 \%$ & $11 \%$ \\
Calcita & $6 \%$ & $1 \%$ & $0 \%$ & $1 \%$ \\
Limolita & $1 \%$ & $0 \%$ & $0 \%$ & $0 \%$ \\
Riolita silicificada & $5 \%$ & $0 \%$ & $0 \%$ & $2 \%$ \\
Rta & $1 \%$ & $0 \%$ & $0 \%$ & $0 \%$ \\
Toba & $1 \%$ & $0 \%$ & $0 \%$ & $0 \%$ \\
Toba silicificada & $4 \%$ & $0 \%$ & $0 \%$ & $0 \%$ \\
Cuarcita & $0 \%$ & $0 \%$ & $0 \%$ & $1 \%$ \\
Xilópalo & $1 \%$ & $0 \%$ & $0 \%$ & $1 \%$ \\
Total & $58 \%$ & $8 \%$ & $1 \%$ & $33 \%$ \\
\hline
\end{tabular}

Cuadro 11: Representación de los tipos de talón de Cueva del Milodón Norte 1 (Santa Cruz)

\begin{tabular}{lccccccc}
\hline \multicolumn{1}{c}{ Materia prima } & Cortical & Facetado & Filiforme & Diedro & Astillado & Liso & Punti. \\
\hline Obsidiana & $1 \%$ & $8 \%$ & $11 \%$ & $2 \%$ & $3 \%$ & $6 \%$ & $2 \%$ \\
Basalto / And. & $0 \%$ & $1 \%$ & $3 \%$ & $1 \%$ & $0 \%$ & $5 \%$ & $0 \%$ \\
Silice & $0 \%$ & $7 \%$ & $8 \%$ & $1 \%$ & $0 \%$ & $12 \%$ & $1 \%$ \\
Calcita & $0 \%$ & $1 \%$ & $3 \%$ & $0 \%$ & $0 \%$ & $4 \%$ & $0 \%$ \\
Limolita & $0 \%$ & $0 \%$ & $0 \%$ & $0 \%$ & $0 \%$ & $2 \%$ & $0 \%$ \\
Riolita silicificada & $0 \%$ & $1 \%$ & $1 \%$ & $0 \%$ & $0 \%$ & $5 \%$ & $0 \%$ \\
Rta & $0 \%$ & $0 \%$ & $0 \%$ & $0 \%$ & $0 \%$ & $1 \%$ & $0 \%$ \\
Toba & $0 \%$ & $0 \%$ & $0 \%$ & $0 \%$ & $0 \%$ & $1 \%$ & $0 \%$ \\
Toba silicificada & $0 \%$ & $0 \%$ & $1 \%$ & $0 \%$ & $0 \%$ & $3 \%$ & $0 \%$ \\
Cuarcita & $0 \%$ & $0 \%$ & $0 \%$ & $0 \%$ & $0 \%$ & $1 \%$ & $0 \%$ \\
Xilópalo & $0 \%$ & $0 \%$ & $0 \%$ & $0 \%$ & $0 \%$ & $1 \%$ & $0 \%$ \\
Total & $1 \%$ & $19 \%$ & $27 \%$ & $4 \%$ & $4 \%$ & $41 \%$ & $4 \%$ \\
\hline
\end{tabular}

minaciones y el tipo de lasca u hoja ya que, en la talla bifacial predominan los talones facetados o filiformes. Cabe destacar que en TA el 90,17\% de la muestra corresponde a lascas de reducción primaria y el 9,83\% restante se divide entre lascas de reactivación, talla bifacial y otro origen (sensu Aschero 1975, 1983). Mientras que en Quil 
Cuadro 12: Porcentajes de tipos de terminaciones en desechos de talla de Bajo de la Laguna 2 (Santa Cruz)

\begin{tabular}{lrrrrr}
\hline & Aguda & Charnela & Quebrada & Fractura & Sobrep. \\
\hline Limolita & $0,25 \%$ & $0,00 \%$ & $0,00 \%$ & $0,17 \%$ & $0,00 \%$ \\
Cuarcita & $0,08 \%$ & $0,00 \%$ & $0,00 \%$ & $0,42 \%$ & $0,00 \%$ \\
Obsdiana & $27,30 \%$ & $2,50 \%$ & $1,09 \%$ & $17,45 \%$ & $0,08 \%$ \\
Silice & $18,95 \%$ & $1,25 \%$ & $0,08 \%$ & $15,11 \%$ & $0,00 \%$ \\
Basalto & $1,42 \%$ & $0,17 \%$ & $0,00 \%$ & $1,00 \%$ & $0,00 \%$ \\
Xilopalo & $0,25 \%$ & $0,00 \%$ & $0,00 \%$ & $0,08 \%$ & $0,00 \%$ \\
Calcedonia & $3,51 \%$ & $0,42 \%$ & $0,00 \%$ & $2,92 \%$ & $0,00 \%$ \\
INDI & $0,08 \%$ & $0,00 \%$ & $0,00 \%$ & $0,08 \%$ & $0,00 \%$ \\
Riolita & $1,34 \%$ & $0,00 \%$ & $0,00 \%$ & $1,25 \%$ & $0,00 \%$ \\
Dacita & $0,08 \%$ & $0,08 \%$ & $0,00 \%$ & $0,00 \%$ & $0,00 \%$ \\
Riolita silicificada & $1,34 \%$ & $0,17 \%$ & $0,00 \%$ & $0,67 \%$ & $0,00 \%$ \\
Toba & $0,25 \%$ & $0,00 \%$ & $0,00 \%$ & $0,17 \%$ & $0,00 \%$ \\
Total & $54,84 \%$ & $4,59 \%$ & $1,17 \%$ & $39,32 \%$ & $0,08 \%$ \\
\hline
\end{tabular}

Cuadro 13: Intervalos de ancho de talón de Bajo de la Laguna 2 (Santa Cruz)

\begin{tabular}{lrrrrrrc}
\hline \multirow{2}{*}{ Materia prima } & \multicolumn{7}{c}{ Intervalos } \\
\cline { 2 - 8 } & $\mathbf{1 - 4}$ & $\mathbf{5 - 8}$ & $\mathbf{9 - 1 2}$ & $\mathbf{1 3 - 1 5}$ & $\mathbf{1 6 - 1 9}$ & $\mathbf{2 0 - 2 3}$ & $>\mathbf{2 3}$ \\
\hline Limolita & $0,17 \%$ & $0,17 \%$ & $0,08 \%$ & $0,00 \%$ & $0,00 \%$ & $0,00 \%$ & $0,00 \%$ \\
Cuarcita & $0,34 \%$ & $0,17 \%$ & $0,00 \%$ & $0,00 \%$ & $0,00 \%$ & $0,00 \%$ & $0,00 \%$ \\
Obsdiana & $32,35 \%$ & $13,95 \%$ & $1,43 \%$ & $0,25 \%$ & $0,25 \%$ & $0,08 \%$ & $0,08 \%$ \\
Silice & $24,37 \%$ & $9,08 \%$ & $1,34 \%$ & $0,34 \%$ & $0,17 \%$ & $0,17 \%$ & $0,17 \%$ \\
Basalto & $0,76 \%$ & $1,34 \%$ & $0,25 \%$ & $0,00 \%$ & $0,00 \%$ & $0,00 \%$ & $0,08 \%$ \\
Xilopalo & $0,25 \%$ & $0,08 \%$ & $0,00 \%$ & $0,00 \%$ & $0,00 \%$ & $0,00 \%$ & $0,00 \%$ \\
Calcedonia & $4,12 \%$ & $2,27 \%$ & $0,34 \%$ & $0,08 \%$ & $0,00 \%$ & $0,00 \%$ & $0,00 \%$ \\
INDI & $0,08 \%$ & $0,08 \%$ & $0,00 \%$ & $0,00 \%$ & $0,00 \%$ & $0,00 \%$ & $0,00 \%$ \\
Riolita & $1,43 \%$ & $0,67 \%$ & $0,17 \%$ & $0,00 \%$ & $0,17 \%$ & $0,00 \%$ & $0,08 \%$ \\
Dacita & $0,08 \%$ & $0,08 \%$ & $0,00 \%$ & $0,00 \%$ & $0,00 \%$ & $0,00 \%$ & $0,00 \%$ \\
Riolita silicificada & $0,84 \%$ & $1,01 \%$ & $0,08 \%$ & $0,00 \%$ & $0,00 \%$ & $0,25 \%$ & $0,00 \%$ \\
Toba & $0,17 \%$ & $0,25 \%$ & $0,00 \%$ & $0,00 \%$ & $0,00 \%$ & $0,00 \%$ & $0,00 \%$ \\
Total & $64,96 \%$ & $29,16 \%$ & $3,70 \%$ & $0,67 \%$ & $0,59 \%$ & $0,50 \%$ & $0,42 \%$ \\
\hline
\end{tabular}

5 el $89,81 \%$ corresponde a la reducción primaria y el 10,18\% restante corresponde a lascas de reactivación, talla bifacial, presión y talla tanto en TA como en Quil 5 estas dos variables se encuentran relacionadas, ya que el material que presenta bulbos dobles, en la mayoría de los casos presenta marcas de impacto en las superficies de percusión. En cuanto a las materias primas utilizadas en las que predominan los supuestos errores serían las materias primas más disponibles en las inmediaciones (rocas silíceas y basaltos).

Retomando lo expuesto anteriormente, las colecciones experimentales fueron creadas con un propósito, el de observar evidencias de habilidades diferentes para tallar, y cada una de las lascas producto de esas experiencias, fueron separadas y analizadas. Entonces, el origen de los conjuntos analizados también pudo influir en los resultados obtenidos. Algo que no debe dejar de tenerse en cuenta durante el análisis ya que, los procesos postdepositacionales en los sitios, las formas de recolección de los materiales y, como se mencionara más arriba, el acceso a la materia prima pueden influir en las colecciones arqueológicas analizadas. Una diferencia que podría hacerse, como plantean 
Stout (2002), Reynolds (2011) y otros autores, con el trabajo experimental, es que en él, los talladores no tuvieron problemas de acceso a la materia prima. Stout (2002) plantea que, «debemos preguntarnos cuán selectivos fueron los talladores y cuáles fueron las condiciones y los problemas que tuvieron en localizar, identificar y explotar las materias primas que deseaban» (Stout 2002: 704). Para los aprendices, o para los más inexpertos o menos hábiles, debía volverse más difícil. Por eso consideramos que trabajarían en materia prima de origen local o inmediatamente disponible. En el material analizado, la mayor recurrencia de errores en los desechos y en núcleos sustenta esta idea. Una última cuestión a tener en cuenta acerca de las habilidades técnicas y los talladores es que debemos considerar que la variabilidad que observamos en los conjuntos puede deberse a habilidad o a estilos personales diferentes, como por ejemplo lo planteado por Weedman (2002), entre otros autores. Entonces, cuando realizamos los análisis de material y observamos conjuntos que se definen como «toscos» y otros que se definen como «precisos» ¿qué estamos definiendo? Considero que aquí el trabajo realizado en experimentación (Apel 2008, entre otros) y las observaciones etnoarqueológicas (Stout 2002; Weedman 2002, entre otros) ayudan a entender que, como plantea Nami (1994), «en las sociedades cazadoras-recolectoras, se puede notar que la transmisión del conocimiento empírico por los mecanismos de información, encierra no sólo los transmitidos por la palabra, sino también por la observación y participación interactiva de los miembros del grupo que comparte un paradigma tecnológico. Podemos encontrar innumerables referencias acerca de cómo las técnicas se transmiten de mayores a menores mediante juegos, observación, y otros medios de comunicación humana oral y gestual». Aquí es donde entra en circulación la información en un nivel micro, o, como plantea Gamble (1993a, 1993b, 1998, 1999) en la red íntima. Esta red formada por el grupo más cercano sería en la que se produciría este tipo de intercambio, y representa la primera red de seguridad de los individuos, en ella se obtiene la asistencia material y emocional necesaria para el desarrollo. Aprender y enseñar son fundamentales a la adaptación humana, a la socialización, al cambio cultural y en un nivel más amplio a la producción y reproducción de cultura en una sociedad (Pelissier 1991).

Pero más allá del análisis puramente material, la experiencia del aprendizaje generaría, en estos grupos, una idea de comunidad (Lave y Wenger 1991). En este sentido, aprendizaje se desarrolla, en la mayoría de los casos, en el transcurso de las actividades cotidianas. Las técnicas entonces, así como las normas y los roles, son aprendidas en el hacer, en el transcurso de lo cotidiano (Pelissier 1991).

Se vuelve interesante cómo a través del trabajo experimental, sumado a un análisis minucioso de los desechos de talla y los núcleos, se puede acceder a ciertas formas de interacción del pasado que, de otro modo, podrían pasar inadvertidas.

\section{Consideraciones finales}

En este trabajo se buscó realizar un aporte al estudio de los grupos cazadores-recolectores que habitaron la Patagonia centro meridional. Principalmente, el trabajo se orientó a identificar, en lo que Torrence (2001) llama micro-escala, las posibles variaciones entre talladores y evidencias que permitieran, en el futuro, identificar 
diferentes habilidades para la talla. El objetivo último era buscar indicadores de lo que Gamble (1993a, 1993b, 1998 y 1999) llamó red intima y red eficaz, pensando en la interacción entre los talladores y en los contextos de enseñanza-aprendizaje. Por supuesto que se tuvo en cuenta lo promediado del registro (Borrero 2001) con el que se trabaja y no se pretendió en ningún momento hacer analogías.

En el análisis de la micro-escala, sin embargo, no debe dejar de tenerse en cuenta que en sitios donde la materia prima de buena a mediana calidad para la talla se encuentre disponible en las cercanías, la actividad de talla expeditiva por expertos talladores puede incluso generar resultados similares a los observados para talladores menos hábiles. Esto no inhabilita la discusión en los casos de estudio analizados ya que se tomó la precaución de pensar en los casos de equifinalidad.

Por otro lado, al comparar los resultados obtenidos de los sitios arqueológicos analizados, y luego su comparación con el material experimental surgieron algunos puntos que deben ser destacados. Los sitios de actividades múltiples, que presentan mayor reutilización, como es el caso de los sitios de Chubut y CMN1, mostraron mayores evidencias de errores de talla. Mientras que BLG2 presenta niveles muy bajos, casi nulos. Esto podría deberse a que este sitio cumplió funciones diferentes a los anteriores. La ubicación de las fracturas observadas en los fragmentos de puntas de proyectil, sumadas a la alta representación de fragmentos de filo y raspadores, podrían indicarnos un sitio de actividades limitadas, probablemente orientado al recambio y mantenimiento de puntas de proyectil (Sacchi 2013). Es esperable encontrar evidencias de «aprendizaje» o de talladores menos hábiles en los sitios de actividades múltiples, que podrían corresponder a campamentos base y no en sitios de actividades limitadas.

Otro punto destacable es la ampliación del término talladores inexpertos discutiéndolo en base a la posibilidad de identificación de habilidades o pericia para la actividad de talla. Aquí fue fundamental el análisis del índice de eficiencia para la talla en el caso de los talladores experimentales y luego el posterior análisis en el material arqueológico. Como se mencionara anteriormente, el trabajo experimental permitió generar hipótesis y expectativas que fueron contrastadas en el registro arqueológico. A partir de estos análisis pudieron identificarse variables que permitieron inferir diferentes habilidades para la talla lítica y aportar a la discusión sobre la funcionalidad de los sitios.

Agradecimientos: Este trabajo fue posible gracias al financiamiento de la U.B.A a través de una beca doctoral, el CONICET y la ANPCyT. Por otro lado debo agradecer a mi directora Dra. Cecilia Pérez de Micou, a mis compañeras de equipo y campañas en el Chubut. A María Teresa Civalero por guiarme y llevarme por la senda del análisis lítico. A todo el equipo de trabajo de Santa Cruz: Carlos Aschero, Mariana De Nigris, Damián Bozzuto, Natalia Fernández, Sofía Tecce y Luis Horta. A la familia Otamendi y a Mario Espona por el apoyo recibido en la Estancia Pueyrredón. A las familias Pérez del Barrio y Solsona, por el apoyo y la ayuda brindada en Aldea Beleiro. A Carlos y Cristina Palenque que nos salvan cada vez que vamos a Comodoro. Finalmente, todo lo expuesto aquí es de mi estricta responsabilidad. 


\section{Referencias bibliográficas}

APEL, Jan

2008 «Knowledge, Know-How and Raw Material - The Production of Late Neolithic Flint Daggers in Scandinavia». Journal of Archaeological Method and Theory 15 (1): 91-111.

Aschero, Carlos Alberto

1975 «Ensayo para una clasificación morfológica de artefactos líticos aplicada a estudios tipológicos comparativos». Informe depositado en el CONICET.

1983 «Ensayo para una clasificación morfológica de artefactos líticos. Apéndice A y B». Cátedra de Ergología y Tecnología. Facultad de Filosofía y Letras, Universidad de Buenos Aires.

Aschero, Carlos Alberto, Damián Bozzuto, María Teresa Civalero, Mariana De Nigris, Antonela Di Vruno, Vanina Dolce, Natalia Fernández, Laura González y Paula LIMBRUNNER

2009 «El registro arqueológico de la costa noreste del Lago Pueyrredon-Cochrane (Santa Cruz, Argentina)», en Arqueología de Patagonia: una mirada desde el último confin, Mónica Salemme et al., eds., pp. 919-926. Ushuaia: Editorial Utopías.

Aschero, Carlos Alberto, Damián Bozzuto, María Teresa Civalero, Mariana De Nigris, Natalia Fernández, Luis Rubén Horta, Adriana Lacrouts, Mariana Sacchi y Sofía Tecce 2010 «Ocupaciones humanas en la costa norte del Lago Pueyrredón y el área de Laguna de los Cisnes: avances en las investigaciones». Ponencia presentada en el XVII Congreso Nacional de Arqueología Argentina.

Aschero, Carlos Alberto, Mariana De Nigris, María José Figuerero Torres, Ana Gabriela Guráieb, Guillermo Mengoni GoÑalons y Hugo Yacobaccio

1999 «Excavaciones recientes en Cerro de los Indios 1, Lago Posadas (Santa Cruz): nuevas perspectivas», en Soplando en el viento... Actas de las III Jornadas de Arqueología de la Patagonia, pp. 269-286. Buenos Aires - Neuquén: INAPL Universidad Nacional del Comahue.

Bodu, Pierre, Claudine Karlin y Silvie Ploux

1990 «Who is Who? The Magdalenian Flintknappers of Pincevent, France», en The Big Puzzle, Erwin Cziesla et al., eds., pp. 143-163. Bonn: Holos.

BORRERO, Luis Alberto

1994-95 «Arqueología de la Patagonia». Palimpsesto. Revista de Arqueología 4: 9-69.

2001 «Regional Taphonomy: The Scales of Application to the Archaeological Record», en Animals and Man in the Past, H. Buitenhuis y W. Prummel, eds., pp. 17-20. Groningen: ARC Publications.

Bozzuto, Damián, Luis Rubén HorTa y Mariana SACCHI

2012 «Variación en los niveles lacustres de la Cuenca Pueyrredón-Cochrane: circulación de materias primas en la Costa NE del Lago Pueyrredón». Ponencia presentada en el $54^{\circ}$ Congreso Internacional de Americanistas, Viena.

Bozzuto, Damián, Mariana Sacchi, Luis Rubén Horta, María Teresa Civalero y Carlos ASCHERO

2013 «Circulación de Materias primas y movilidad: Influencia de las variaciones en los niveles lacustres de la cuenca Pueyrredón-Cochrane». Ponencia presentada en el XVIII Congreso Nacional de Arqueología Argentina, La Rioja. 
Castro Esnal, Analía

2010 Rutas indígenas y arqueología en la provincia de Chubut. Tesis de Doctorado. Facultad de Filosofía y Letras, Universidad de Buenos Aires.

Castro Esnal, Analía, Mariana Sacchi y Cecilia Pérez de Micou

2011 «Aspectos generales de la tecnología lítica del sitio de la Colonia El Chalía SO de la provincia de Chubut, Argentina». International Journal of South American Archaeology 9: 28-40.

De Nigris, Mariana, María José Figuerero Torres, Ana Gabriela Guráieb y Guillermo Mengoni GoÑalons

2004 «Nuevos fechados radiocarbónicos de la localidad de Cerro de los Indios 1 (Santa Cruz) y su proyección areal», en Contra viento y marea. Arqueología de Patagonia, $\mathrm{M}^{\mathrm{a}}$ Teresa Civalero et al., eds., pp. 537-544. Buenos Aires: Instituto Nacional de Antropología y Pensamiento Latinoamericano.

DoBres, Marcia-Ann

1999 «Technology's Links and Châines: The Processual Unfolding of Technique and Technician», en The Social Dynamics of Technology: Practice, Politics, and World Views, Marcia-Ann Dobres y Christopher R. Hoffman, eds., pp. 124-146. Washington: Smithsonian Institution Press.

ERICSON, Jonathon E.

1984 «Toward the Analysis of the Lithic Production Systems», en Prehistoric Quarries and Lithic Production in Archaeology, Jonathon E. Ericson y Barbara A. Purdy, eds., pp. 1-10. Cambridge: Cambridge University Press.

FINLAY, Nyree

1997 «Kid Camping: The Missing Children in Lithic Analysis», en Invisible People and Processes. Writing Gender and Childhood into European Archaeology, Jenny Moore y Eleanor Scott, eds., pp. 203-212. Londres: Leicester University Press.

Galarce, Patricio

2008 «Aprendizaje y talla lítica en sociedades prehistóricas: contextos sociales y correlatos materiales», en Puentes hacia el pasado. Reflexiones teóricas en Arqueología, Donald Jackson et al., eds., pp. 93-111. Santiago de Chile: Sociedad Chilena de Arqueología.

Gamble, Clive

1993a «Exchange, Foraging and Local Hominid Networks», en Trade and Exchange in Prehistoric Europe, Christopher. Scarre y Frances Healy, eds., pp 35-44. Oxford: Oxbow Books.

1993b «People on the Move: Interpretations of Regional Variation in Paleolithic Europe», en Cultural Transfomations and Interactions in Eastern Europe, John Chapman y Pavel Dolukhanov, eds., pp. 37-55. Centre for the Archaeology of Central and Eastern Europe Monograph 1. Avebury: Ashgate Publishing.

1998 «Paleolithic Society and the Release from Proximity: A Network Approach to Intimate Relations». World Archaeology 29 (3): 426-449

1999 The Paleolithic Societies of Europe. Cambridge: Cambridge University Press.

GoÑI, Rafael

2000-02 «Fechados radiocarbónicos y registro arqueológico en la cuenca de los lagos Salitroso/Posadas (Santa Cruz)». Cuadernos del Instituto Nacional de Antropología y Pensamiento Latinoamericano 19: 666-668. 
2011 Cambio climático y poblamiento humano durante el Holoceno tardio en Patagonia meridional. Una perspectiva arqueológica. Tesis doctoral. Universidad de Buenos Aires, Facultad de Filosofía y Letras. MS.

GoÑI, Rafael y Gustavo BARRIENTOS

2004 «Poblamiento tardío y movilidad en la cuenca del lago Salitroso», en Contra viento y marea. Arqueología de Patagonia, T. Civalero et al., eds., pp. 313-324. Buenos Aires: INAPL-SAA.

GoÑI, Rafael, Gustavo BARrientos y Gisela CASSIODORO

2000-02 «Condiciones previas a la extinción de las poblaciones humanas del sur de Patagonia: una discusión a partir del análisis del registro arqueológico de la cuenca del lago Salitroso». Cuadernos del Instituto Nacional de Antropología y Pensamiento Latinoamericano 19: 249-266.

GRIMM, Linda

2000 «Apprentice Flintknapping. Relating Material Culture and Social Practice in the Upper Paleolithic», en Children and Material Culture, Joanna Sofaer Derevenski, ed., pp. 53-71. Londres: Routledge.

Hocsman, Salomón

2006 «Producción de bifaces y aprendices en el sitio Quebrada Seca 3 -Antofagasta de la Sierra, Catamarca (5500-4500 años A.P)», en Procesos sociales prehispánicos en los Andes meridionales, Axel E. Nielsen et al., comps., pp. 55-82. Córdoba: Editorial Brujas.

Karlin, Carline y Michèle JuLIEN

1994 «Prehistoric Technology: A Cognitive Science?», en The Ancient Mind: Elements of Cognitive Archaeology, Colin Renfrew y Ezra B. Zubrow, eds., pp. 152-164. Cambridge: Cambridge University Press.

KuHN, Stephen L.

2004 «Upper Paleolithic Raw Material Economies at Ücazig Cave, Turkey». Journal of Anthropological Archaeology 23: 431-448.

LAVE, Jean y Etienne WeNGER

1991 Situated Learning: Legitimate Peripheral Participation. Cambridge: Cambridge University Press.

McCALl, Grant S., ed.

2011 Pushing the Envelope. Experimental Directions in the Archaeology of Stone Tools. Nueva York: Nova.

Mena, Francisco, Víctor Lucero, Omar Reyes, Valentina Trejo y Héctor Velazquez

2000 «Cazadores tempranos y tardíos en la Cueva Baño Nuevo 1, margen occidental de la estepa centropatagónica (XI Región de Aisén, Chile)». Anales del Instituto de la Patagonia, Serie Ciencias Humanas (Chile) 28: 173-195.

Mengoni Goñalons, Guillermo y Hugo Yacobaccio

2000 «Arqueología de Cerro de los Indios y su entorno». Arqueología 10: 193-214.

Mengoni Goñalons, Guillermo, María José Figuerero Torres, M. Victoria Fernández y Pamela V. CHÁveZ

2009 «Carácter de las ocupaciones humanas en el área de Los Antiguos-Monte Zeballos 
y Paso Roballos (Santa Cruz, Argentina)», en Arqueología de Patagonia: una mirada desde el último confin, Mónica Salemme et al., eds., pp. 1061-1074. Ushuaia: Editorial Utopías.

NAMI, Hugo

1994 «Paleoindio, cazadores-recolectores y tecnología lítica en el extremo sur de Sudamérica continental», en Arqueología de cazadores-recolectores. Límites, casos y aperturas, José Luis Lanata y Luis Alberto Borrero, comps., pp. 89-103. Arqueología Contemporánea, volumen 5, edición especial. Buenos Aires: CONICET.

PELISSIER, Catherine

1991 «The Anthropology of Teaching and Learning». Annual Review of Anthropology 20: 75-95.

PÉrez de Micou, Cecilia, Analía CASTro y Mariana SACCHI

2012 «Estudios preliminares en el sitio Casa de Piedra, Estancia Roselló, sudoeste de Chubut». Libro de Resúmenes, VIII Jornadas de Arqueología de la Patagonia. Malargüe.

Pérez de Micou, Cecilia, Mariana SACChi, Analía CAStro y Luz Funes

2008 «Estudios de arqueología en la Colonia indígena de Chalía, Dpto. Senguerr, Chu-

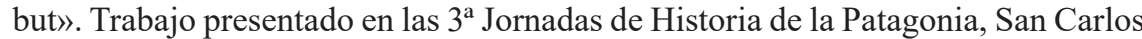
de Bariloche.

Pigeot, Nicole

1990 «Technical and Social Actors. Flintknapping Specialist and Apprentices at Magdalenian Etiolles». Archaeological Review from Cambridge 9 (1): 126-141.

ReYNOLDS, Cerisa R.

2011 «Searching for Skill Identifiers Through Experimental Flintknappig and a North American Archaeolgical Assemblange», en Pushing the Envelope: Experimental Directions in the Archaeology of Stones Tools, Grant S. McCall, ed., pp. 19-38. Nueva York: Nova.

SACCHI, Mariana

2009a «Al maestro con cariño. Identificando aprendices en el registro arqueológico», en Entre pasados y presentes II. Estudios contemporáneos en Ciencias Antropológicas, Tirso Bourlot et al., eds., pp. 155-170. Buenos Aires: Fundación de Historia Natural Félix de Azara.

2009b «Tallando piedras, salvando errores: análisis de desechos de talla experimentales», en Arqueología de Patagonia: una mirada desde el último confín, Mónica Salemme et al., eds., pp. 383-392. Ushuaia: Editorial Utopías.

2010 «Exploración de variables de análisis experimentales aplicadas a material arqueológico: identificación de marcadores de pericia técnica en la talla lítica», en Arqueología argentina en el bicentenario de la Revolución de Mayo, J. Roberto Bárcena y Horacio Chiavazza, eds., tomo 1, pp. 49-55. Mendoza: Universidad Nacional de Cuyo - CONICET.

2012 Materias primas y redes sociales en los grupos cazadores-recolectores de Patagonia Centro Meridional. Tesis Doctoral Inédita. Buenos Aires, Facultad de Filosofía y Letras, Universidad de Buenos Aires.

2013 «A un paso de la laguna. Análisis lítico del sitio Bajo de la Laguna 2, provincia de Santa Cruz». Comechingonia Virtual 7 (2): 216-233.

2014 «Consideraciones preliminares sobre indicadores de pericia técnica en núcleos y artefactos producidos por talla bifacial. Una aproximación experimental». Intersecciones en Antropología 15: 323-337. 
Sacchi, Mariana, Damián Bozzuto, Luis Horta, Natalia Fernández, Mariana De Nigris, Teresa Civalero y Carlos Aschero

2016 «Dataciones y circulación humana: influencia de las fluctuaciones del sistema lacustre Pueyrredón Posadas durante el Holoceno». Revista Andes 27. http://www. redalyc.org/articulo.oa?id=12749260011

SOFFER, Olga

1991 «Lithics and Lifeways - The Diversity in Raw Material Procurement and Settlement Systems on the Upper Paleolithic East European Plain», en Raw Material Economies among Prehistoric Hunter-Gatherers, Anta Monet-White y Steven Holen, eds., pp. 221-234. Lawrence: University of Kansas.

STINe, Scott

1994 «Extreme and Persistent Drought in California and Patagonia During Mediaeval Time». Nature 369: 546-549.

2000 «On the Medieval Climatic Anomaly». Current Anthropology 41 (4): 627-628.

STINE, Scott y Mary STINE

1990 «A Record from Lake Cardiel of Climate Change in Southern America». Nature 345: 705-708.

STOUT, Dietrich

2002 «Skill and Cognition in Stone Tool Production. An Ethnographic Case Study from Irian Jaya». Current Anthropology 43 (5): 693-721.

TORRENCE, Robin

2001 «Hunter-Gatherer Technology: Macro- and Microscale Approaches», en HunterGatherers: An Interdisciplinary Perspective, Catherine Panter-Brick et al., eds., pp. 73-98. Cambridge: Cambridge University Press.

WeEDMan, Kathryn J.

2002 «On the Spur of the Moment: Effects of Age and Experience on Hafted Stone Scraper Morphology». American Antiquity 67 (4): 731-744.

WhaLlON, Robert

2006 «Social Networks and Information: Non-'utilitarian’ Mobility Among HunterGatherers». Journal of Anthropological Archaeology 25: 259-270.

WhitTAKer, John C.

1994 Flintknapping. Making and Understanding Stone Tools. Austin: University of Texas Press.

Yacobaccio, Hugo y A. G. Guráieb

1994 «Tendencia temporal de contextos arqueológicos: área del río Pinturas y zonas vecinas», en Contribución a la arqueología del río Pinturas, provincia de Santa Cruz, Carlos J. Gradín y Ana M. Aguerre, eds., pp. 13-28. Concepción del Uruguay: Búsqueda de Ayllu. 